\title{
Signatures of supernova neutrino oscillations into extra dimensions
}

\author{
G. Cacciapaglia and M. Cirelli \\ Scuola Normale Superiore and INFN Sezione di Pisa, Piazza dei Cavalieri 7, I-56126 Pisa, Italy
}

A. Romanino

Theory Division, CERN, CH-1211 Geneva, Switzerland

(Received 7 March 2003; published 27 August 2003)

\begin{abstract}
We consider the mixing of muon and tau neutrinos with sterile fermion fields propagating in extra dimensions in the context of core collapse supernova physics, extending the analysis of the electron neutrino case done in a previous work. We show that the potentially dramatic modifications to the supernova evolution are prevented by a mechanism of feedback, so that no severe bounds on the parameters of the extra dimensions need to be imposed. Nevertheless, the supernova core evolution is significantly modified. We discuss the consequences on the delayed explosion mechanism and the compatibility with the SN 1987A signal. Then, for the cases of both $\nu_{\mu, \tau}$ and $\nu_{e}$ mixing with bulk fermions, we analyze the distinctive features of the signal on Earth.
\end{abstract}

DOI: $10.1103 /$ PhysRevD.68.033013

PACS number(s): 14.60.St, 97.60.Bw

\section{INTRODUCTION}

The study of sterile neutrinos propagating in the bulk of extra space dimensions [1] and of core collapse supernova evolution [2] establishes a nice link that can have interesting implications on both sides, even though the role of sterile neutrinos is not believed to be primary in solar and atmospheric contexts $[3,4]$. We consider indeed a framework [5] in which the solar and atmospheric oscillation signals are independently accounted for in the standard way. In addition, a small mixing with the 4-dimensional Kaluza-Klein (KK) states (the 4D correspondent of the extra dimensional field) is the source of the nonstandard effects on supernova ( $\mathrm{SN}$ ) physics, since it implies resonant oscillations with ordinary Standard Model neutrinos via matter effects. The point is that we have a large number of resonances that can enhance the effect, even if the mixing angles are small. As KK modes do not interact with ordinary matter and are therefore not trapped, conversions lead to a faster cooling and deleptonization of the SN dense core. The observed SN 1987A signal [6] requires such an energy loss not to take place with a time scale much shorter than $10 \mathrm{sec}$. However, the consequent potentially severe bounds on the extra dimension size [7] are widely relaxed by a feedback mechanism [8,9] that selflimits the conversion. In a previous work [9], we analyzed the possibility of a mixing between the electron neutrinos and a sterile bulk fermion and we explicitly showed that the mechanism indeed prevents the potentially dramatic cooling in a wide portion of the parameter space. We also pointed out that new interesting effects on the SN dynamics can arise in the enlarged parameter space. We speculated that the enrichment of the $\nu_{e}$ component in the outgoing neutrino flux might help the delayed shock mechanism and drive the explosion. ${ }^{1}$ Furthermore, we found that while this picture is

\footnotetext{
${ }^{1}$ The difficulty to obtain an explosion in simulations, however, can be explained by the complexity of the system to be simulated, so that no new physics is in principle necessary.
}

in good agreement with the observed SN 1987A signal, the flux composition can be significantly modified.

In the first part of the present work we address the case of muon and tau neutrinos. In the standard picture of collapse and cooling of the $\mathrm{SN}$ core, their role is rather different from the electron ones. In fact, while electron neutrinos are copiously produced by the neutronization process during collapse and build up a degenerate $\nu_{e}$ sea with large chemical potential $\mu_{\nu_{e}}$ (therefore electron antineutrinos are a negligible fraction in these conditions), muon and tau neutrinos and antineutrinos are produced in pairs and have almost vanishing chemical potential. Moreover, lacking in charged current interactions, they have a faster diffusion and give an important contribution to the transport of thermal energy out of the core. Also, their lepton number does not play a significant role in the diffusion dynamics.

The mixing of tau or muon neutrinos with bulk fermions opens an unconventional escape channel, which can affect the SN core cooling and deleptonization in a peculiar way. We will see that the usual energy loss bounds can be relaxed, as in the electron case, by the action of a feedback. We will then be interested in the phenomenological consequences on the revival of the shock and in the implications for the SN 1987A signal.

In the last part of the paper we discuss the peculiar signatures of the considered effects on the neutrino signal on the Earth. This we do not only for the case of $\nu_{\mu}, \nu_{\tau}$ but also for the case of $\nu_{e}$ conversion considered in [9].

In the following we will assume that the sterile fermions mix predominantly with the tau neutrino. This is the case if the SM/bulk neutrino couplings reflect the hierarchical structure of the SM Yukawa couplings. Actually, the inclusion of a significant muon neutrino mixing with bulk fields would just unnecessarily complicate the core dynamics. The point is that the oscillations into bulk neutrinos build up a nonzero tau (or muon) lepton number. The tau neutrino abundance is simply determined by the tau lepton number, since tau leptons are not thermally produced, as their mass is large with respect to the energies involved $(T \sim 30 \mathrm{MeV})$. On the con- 
trary, the muon neutrino abundance would also depend on the non-negligible muon fraction through the $\beta$-equilibrium processes $\mu^{-} p \leftrightarrow n \nu_{\mu}$ and $\mu^{+} n \leftrightarrow p \bar{\nu}_{\mu}$. One can also wonder whether the $\nu_{\mu}-\nu_{\tau}$ mixing could reintroduce the muon fraction in the problem. This is not the case, since that mixing is largely suppressed by the difference at one loop of the muon and tau matter potential [10] and by an even larger difference originating from the new effect. In fact, as soon as the resonant conversion into the bulk begins, a nonvanishing tau lepton number is generated and thus the matter interactions produce different effective masses for tau and muon (anti)neutrinos. For sufficiently large mixing with the extra dimension fields, as is our case, the oscillations are suppressed before they significantly influence the neutrino fractions. Notwithstanding the complications described above, we do not expect a qualitatively different behavior in the presence of muons; in Sec. IV we will thus deduce the case of $\nu_{\mu}$ mixing with bulk fermions from our results.

The paper is organized as follows. In Sec. II we briefly describe the general extra dimensional scenario and set the parameter space in which we are interested. In Sec. III we first sketch the expected effect of such new physics on the evolution and then present the numerical model that we use. Finally, in Sec. IV we address the new phenomenology based on the results of our calculation and we compare the expected neutrino flux on Earth in the cases of $\nu_{e}, \nu_{\mu}$, and $\nu_{\tau}$ conversions. In Sec. V we sum up.

\section{FRAMEWORK}

The cornerstones of our large extra dimension scenario are the following. We consider a single gravitational extra dimension, ${ }^{2}$ probed by a sterile fermionic field, which results from a 4D point of view as a KK tower of states, predominantly mixed with tau neutrinos.

The parameter space we are interested in is illustrated in Fig. 1. The radius $R$ of the extra dimension, which sets the scale of the KK spectrum, is allowed to lie in the broad range $10^{-3} \mathrm{eV} \lesssim 1 / R \leqq 1 \mathrm{keV}$. The lower bound comes from direct gravity probes, the upper one from requiring that a sufficient number of resonances be crossed by neutrinos travelling in the core, a condition for observable effects to arise. The mixing angle of the $\nu_{\tau}$ with the $k$ th eigenstate of mass $M_{k}$ is generically parametrized as $\theta_{k} \simeq m / \sqrt{2} M_{k}$, which defines the parameter $m$; we only assume that the density of KK states is proportional to $R$, a feature that is quite model independent. The condition $m R \ll 1$ ensures the smallness of the mixings, while keeping subleading effects under control [9] requires $m R \ll 2 \times 10^{-4}$. The above requests identify the unshaded region in Fig. 1. In this region the new effects enter the analysis only through the crucial parameter $m^{2} R$.

If feedback effects were not taken into account, only values of $m^{2} R$ up to $10^{-10} \mathrm{eV}$ would be compatible with the energy loss argument. For example, for $m^{2} R=5$

\footnotetext{
${ }^{2}$ More extra dimensions could be present at higher energies. This scenario is compatible both with a fundamental gravity scale at $\sim 1 \mathrm{TeV}$ [11] and with astrophysical and cosmological bounds [12].
}

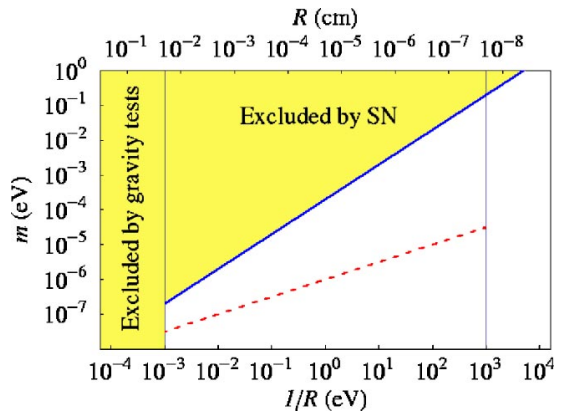

FIG. 1. Excluded regions in the $m-R$ plane, for the case of $\nu_{\tau}$ $\left(\nu_{\mu}\right)$ escape into the bulk. The dashed line (corresponding to $m^{2} R$ $=10^{-12} \mathrm{eV}$ ) marks the lower border of the region where the supernova core evolution is sensibly modified. In the region on the right $(1 / R>1 \mathrm{keV})$ the effect is not active: neutrinos do not cross a sufficient number of resonances.

$\times 10^{-5} \mathrm{eV}$, the core would cool down in about $10^{-5} \mathrm{sec}$. On the contrary, including the feedback, any value of $m^{2} R$ up to $5 \times 10^{-5} \mathrm{eV}$ turns out to be acceptable, while beyond that point the core is cooled by otherwise subleading effects. Thus, a new portion of the parameter space, $10^{-10} \mathrm{eV}$ $\lesssim m^{2} R \leqq 5 \times 10^{-5} \mathrm{eV}$, is opened once the feedback is taken into account. ${ }^{3}$ In the following we will focus on the range

$$
10^{-12} \mathrm{eV} \lesssim m^{2} R \lesssim 5 \times 10^{-5} \mathrm{eV},
$$

where the neutrino escape affects the core evolution in a sizable way.

Tau neutrinos in the SN core experience a MikheyevSmirnov-Wolfenstein (MSW) potential given by

$$
V_{\tau}=\sqrt{2} G_{\mathrm{F}} n_{B}\left(\frac{1}{2} Y_{e}+2 Y_{\nu_{\tau}}+Y_{\nu_{e}}-\frac{1}{2}\right),
$$

where $G_{\mathrm{F}}$ is the Fermi constant, $n_{B}$ is the baryon number density, and $Y_{x}$ is the net number fraction per baryon of the species $x$ : $Y_{x}=\left(N_{x}-N_{x}^{-}\right) / n_{B}$. It will also be useful to consider the lepton number fractions $Y_{L_{e, \mu, \tau}}=Y_{e, \mu, \tau}$ $+Y_{\nu_{e}, \nu_{\mu}, \nu_{\tau}}$

At the beginning $Y_{e}$ is the dominant term and, since it is not expected to be larger than 0.4 in the inner core [2], $V_{\tau}$ is clearly negative. The tau antineutrinos acquire an effective squared mass $m_{\mathrm{eff}}^{2}=2 E_{\nu}^{-} V$, where $E_{\nu}^{-}$is the antineutrino energy. The effective mass changes slightly along the neutrino path: whenever $m_{\text {eff }}$ equals the mass of one of the KK sterile states, a resonance occurs and the tau antineutrino has a certain probability of oscillating into a bulk state and escaping from the core. As a result, the tau lepton number increases and the potential becomes less negative. If the potential were positive the resonances would be met by neutrinos and the potential would get smaller. Note also that $Y_{\nu_{\tau}}$ is zero at the

\footnotetext{
${ }^{3}$ Compared with the electron neutrino escape case, the overall shift to higher values is justified (i) by the smaller number density of $\bar{\nu}_{\tau}$ with respect to $\nu_{e}$, since they are thermal, not degenerate, at the beginning; (ii) marginally, by the fact that $\nu_{\tau}$ diffusion is faster.
} 
beginning but a positive value will be generated by the $\bar{\nu}_{\tau}$ escape itself. This term, which has a different weight for the $\nu_{\mu}$, is responsible for the suppression of the vacuum $\nu_{\mu}-\nu_{\tau}$ oscillations.

The disappearance probability of a $\bar{\nu}_{\tau}$ into the bulk when travelling a distance $L$ is given by the usual Landau-Zener formula

$$
P\left(\bar{\nu}_{\tau} \rightarrow \text { bulk }\right) \simeq L \frac{\pi}{2 \sqrt{2}} m^{2} R\left(\frac{\left|V_{\tau}\right|}{E_{\bar{\nu}}}\right)^{1 / 2}
$$

As is apparent, the parameter $m^{2} R$ sets the overall magnitude of the escape effect.

\section{MODEL AND EVOLUTION}

Having introduced all the ingredients of the problem, let us first discuss the basic features of the expected evolution, compared when necessary with the case of $\nu_{e}$ conversion addressed in [9].

As already mentioned, the matter MSW potential is initially negative in the whole inner core so that only $\bar{\nu}_{\tau}$ cross resonances. As conversions push $V_{\tau}$ towards zero and deleptonization pulls it down to its negative minimum value, a nontrivial interplay between diffusion and the new effect takes place. Nevertheless, the main features of the evolution can be easily understood. First of all, since the new physics is in the $\nu_{\tau}$ sector, the $Y_{L_{e}}$ evolution is only indirectly affected, mainly via the modifications to the temperature. As the $\nu_{e}$ 's are almost degenerate, we expect their diffusion to be only slightly changed and in particular the deleptonization time scale to be unaffected. On the other hand, the $\bar{\nu}_{\tau}$ escape generates a positive $Y_{\nu_{\tau}}$, the balance $\nu_{\tau^{-}} \bar{\nu}_{\tau}$ is broken and a positive chemical potential $\mu_{\nu_{\tau}}$ arises. This in turn inhibits the escape itself, both because $V_{\tau}$ is lifted towards zero by the term $Y_{\nu_{\tau}}$ in Eq. (2) and, more important, because the $\bar{\nu}_{\tau}$ abundance is suppressed in the presence of the chemical potential. In particular, $V_{\tau}$ has no chances to switch to positive values and only $\bar{\nu}_{\tau}$ do convert during all the evolution. Now, how do we expect the $T$ evolution to be affected? In a first fast phase, as long as the conversion is dominant, a portion of the thermal energy is absorbed both by bulk neutrinos and by the sea of increasingly degenerate $\nu_{\tau}$ 's. During all the following evolution, the thermal energy that the $\bar{\nu}_{\tau}$ 's escape keeps on transferring to tau neutrinos is carried outside by their diffusion. Thus, we expect the $T$ evolution to be sped up with respect to the standard case. Moreover, we expect the $\bar{\nu}_{\tau}$ escape cooling channel to be more effective than in [9] as the $\nu_{\tau}$ diffusion time scale is shorter than for $\nu_{e}$ 's. Finally, the tau lepton number that arises in the inner core diffuses out into the mantle and may significantly influence the outgoing flux composition.

To study the above picture in some more detail, we use a simplified model that includes all the relevant physics, although it is not intended as a complete description of the SN core dynamics. A more detailed analysis would require a complete simulation of the core and of the external layers, with the inclusion of the new effect.

We follow all the core evolution in terms of the temperature $T$, the electron leptonic fraction $Y_{L_{e}}$ and the tau leptonic fraction $Y_{L_{\tau}}$ (that is equal to the neutrino fraction $Y_{\nu_{\tau}}$ ). One can express all the other relevant observables (chemical potentials, entropy, fluxes,....) simply as functions of these quantities, using thermodynamic equilibrium, beta equilibrium and charge neutrality. As in [9], we consider a typical $\mathrm{SN}$ core characterized by a mass of $1.5 M_{\odot}$ and a radius of $12.7 \mathrm{~km}$. For the matter density, we use a static profile $\rho(r)$, justified by the relative hydrodynamical stability of the inner core in which we are interested. Explicitly $\rho(r)=\rho_{c} /[1$ $\left.+(r / \bar{r})^{3}\right]$, with $\rho_{c}=7.5 \times 10^{14} \mathrm{~g} / \mathrm{cm}^{3}$ and $4 / 3 \pi \bar{r}^{3} \rho_{c}$ $=1.1 M_{\odot}$. As for the equation of state, we consider a core made only of $n, p, e^{ \pm}, \nu_{e, \mu, \tau}, \bar{\nu}_{e, \mu, \tau}$ and $\gamma$. The effective nucleon mass is expressed as $m_{N}^{*}=m_{N} /\left[\left(1+\beta_{0}\right)\left(\rho / \rho_{0}\right)\right]$, where $m_{N}=939 \mathrm{MeV}$ is the vacuum value, $\beta_{0}$ is chosen to be 0.5 and $\rho_{0}=3 \times 10^{14} \mathrm{~g} / \mathrm{cm}^{3}$ is the reference nuclear density. As for the mean free paths $\lambda_{\nu_{e}}$ and $\lambda_{\nu_{\mu}, \nu_{\tau}}$, in general they would exhibit a nontrivial dependence on the neutrino energy $E_{\nu}$ and the evolution variables $\left(\rho, T, Y_{L_{e}}, Y_{L_{\tau}}\right)$, besides on several other aspects related to the medium in which the neutrinos diffuse. However, the essential features can be grasped by assuming a simple inverse quadratic dependence on the neutrino energy for all species and incorporating an inverse dependence on matter density for muon and tau neutrinos, while keeping constant with density the mean free path of electron neutrinos. We checked these simplified assumptions versus the more complete modellings of [13]. Moreover, the above choices allow us to obtain an evolution whose main features and time scales agree with the results of more sophisticated analyses. The expressions we use are the following: 4

$$
\lambda_{\nu_{e}}\left(E_{\nu}\right)=\lambda_{\nu_{e}}^{0} \frac{E_{\nu, 0}^{2}}{E_{\nu}^{2}}, \quad \lambda_{\nu_{\mu}, \nu_{\tau}}\left(E_{\nu}, r\right)=\lambda_{\nu_{\mu}, \nu_{\tau}}^{0} \frac{\rho_{c}}{\rho(r)} \frac{E_{\nu, 0}^{2}}{E_{\nu}^{2}}
$$

taking $\lambda_{\nu_{e}}^{0}=1.2 \mathrm{~cm}$ and $\lambda_{\nu_{\mu}, \nu_{\tau}}^{0}=2.8 \mathrm{~cm}$ at the reference energy $E_{\nu, 0}=260 \mathrm{MeV}$ and reference density $\rho_{c}=7.5$ $\times 10^{14} \mathrm{~g} / \mathrm{cm}^{3}$. The initial profiles for $T$ and $Y_{L_{e}}$ are typical ones as taken in [9]. Of course, the profile for $Y_{L_{\tau}}$ is zero in all the core at the beginning.

Finally, with all the above prescriptions (see [9] for a more detailed discussion), the evolution equations for $Y_{L_{e}}$, $Y_{L_{\tau}}$, and $T$ read

\footnotetext{
${ }^{4}$ With respect to the electron neutrino case of [9] we refined the value of $\lambda_{\nu_{\mu}, \nu_{\tau}}^{0}$ and we introduced the density dependence for muon and tau neutrinos.
} 


$$
\begin{aligned}
& n_{B} \frac{\partial Y_{L_{e}}}{\partial t}=\vec{\nabla} \cdot\left(a_{e} \vec{\nabla} \mu_{\nu_{e}}\right) \\
& n_{B} \frac{\partial Y_{L_{\tau}}}{\partial t}=\vec{\nabla} \cdot\left(a_{\tau} \vec{\nabla} \mu_{\nu_{\tau}}\right) \\
&+\frac{1}{4 \sqrt{2} \pi} m^{2} R \sqrt{\left|V_{\tau}\right|} T^{5 / 2} F_{3 / 2}\left(-\frac{\mu_{\nu_{\tau}}}{T}\right) \\
& n_{B} T \frac{\partial s}{\partial t}= a_{e}\left(\vec{\nabla} \mu_{\nu_{e}}\right)^{2}+a_{\tau}\left(\vec{\nabla} \mu_{\nu_{\tau}}\right)^{2} \\
&\left.+\vec{\nabla} \cdot\left(a_{e}+a_{\mu}+a_{\tau}\right) \frac{\pi^{2}}{6} \vec{\nabla} T^{2}\right) \\
&-\frac{1}{4 \sqrt{2} \pi} m^{2} R \sqrt{\left|V_{\tau}\right|} T^{7 / 2}\left[F_{5 / 2}\left(-\frac{\mu_{\nu_{\tau}}}{T}\right)\right. \\
&+\frac{\mu_{\nu_{\tau}}}{T} F_{3 / 2}\left(-\frac{\mu_{\nu_{\tau}}}{T}\right),
\end{aligned}
$$

where $a_{i}=\lambda_{\nu_{i}}^{0} E_{\nu, 0}^{2} /\left(6 \pi^{2}\right), i=e, \mu, \tau ; s$ is the entropy per baryon and the Fermi integrals are defined as $F_{q}(y)$ $=\int_{0}^{\infty} d x x^{q} /\left(e^{x-y}+1\right)$.

One can recognize the standard transport equation for electron neutrinos in Eq. (5a), while Eq. (5b) for the tau neutrinos shows the additional escape term that comes from the integration over neutrino energies of the probability in Eq. (3), for $L$ equal to a mean free path. The first line of the temperature Eq. $(5 \mathrm{c})$ features the heating terms $a_{j}\left(\vec{\nabla} \mu_{\nu_{j}}\right)^{2}$ $(j=e, \tau)$ associated with the degradation of the degeneracy energy of neutrinos reaching regions with lower chemical potential. The novelty is the presence of such a term for tau neutrinos also, since they possess a nonvanishing chemical potential now. After the standard temperature diffusion term $\vec{\nabla} \cdot\left(\vec{\nabla} T^{2}\right)$, last comes the contribution from tau antineutrinos escape, which is a cooling term: its first part is related to the energy flux into bulk when an antineutrino is lost, the second one is associated with the (negative) tau lepton number flux into the bulk and thus with the storing of energy in the tau neutrino degenerate sea.

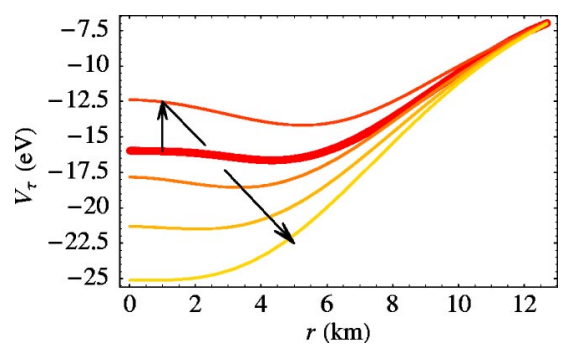

FIG. 2. Profiles of the MSW potential for tau neutrinos. The thick line is the initial configuration corresponding to the typical initial profiles assumed in [9]; the thin lines are snapshots at 1, 5, 10 , and $20 \mathrm{sec}$, following the arrows, for the case $m^{2} R$ $=10^{-7} \mathrm{eV}$.

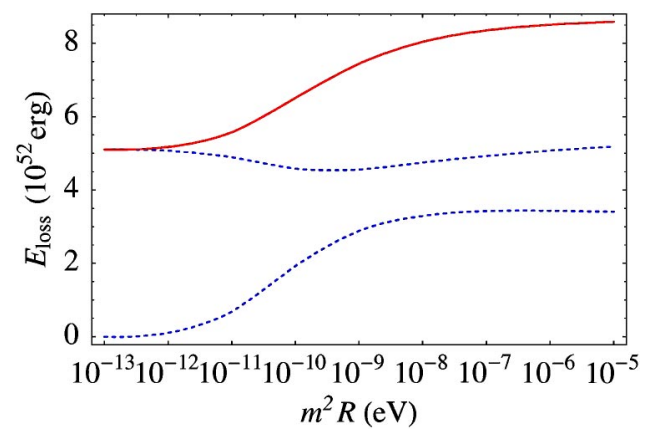

FIG. 3. Energy drained out of the $\mathrm{SN}$ core in the first $10 \mathrm{sec}$. The total energy (solid line) is shown together with its visible (upper dashed line) and invisible (lower dashed line) components as a function of $m^{2} R$.

The numerical solutions of the above equations, for several choices of the parameter $m^{2} R$ in the range of Eq. (1), confirm the expectations presented at the beginning of this section. Essentially, the electron neutrino density follows the standard evolution while the temperature fall is faster. The tau leptonic fraction $Y_{L_{\tau}}$ rapidly grows from zero up to a certain profile (the higher the larger $m^{2} R$, but always below about 0.1 ) and then lowers on typical diffusion time scales. In parallel, the chemical potential $\mu_{\nu_{\tau}}$ quickly grows up to values as large as those of $\mu_{\nu_{e}}$ (of order $200 \mathrm{MeV}$ ) and then clears out with diffusion. A typical evolution of the matter potential is shown in Fig. 2: at the beginning it is pushed towards zero by the rising of $Y_{L_{\tau}}$ but, the initial potential being well below zero, the available energy is not sufficient for a complete zeroing, and diffusion soon reverses the trend.

\section{PHENOMENOLOGY}

Figures 3 and 4 show the energy and the tau lepton number drained out of the inner core in the first $10 \mathrm{sec}$ of evolution, separating the portion that is actually radiated in the visible neutrino flux and the one that is irreparably lost into the invisible channel provided by the bulk. The first $10 \mathrm{sec}$ are the most interesting interval since this is the lapse of time during which the relevant neutrino signal is produced.

As for the energy, one sees that a sizable portion can be lost into the invisible channel, especially for high values of

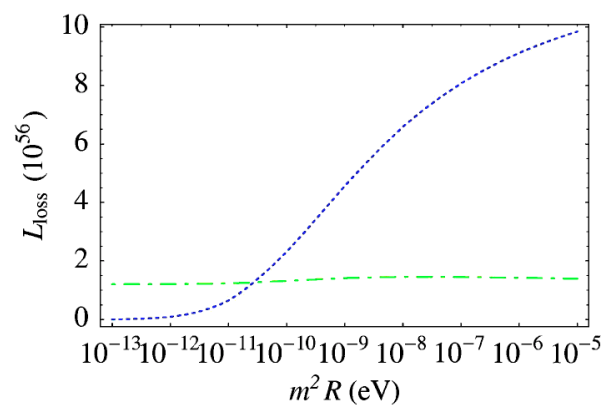

FIG. 4. Tau lepton number emission in the visible channel (dashed line) in the first $10 \mathrm{sec}$. The electron lepton number emission (green dot-dashed line) is also shown for comparison. 


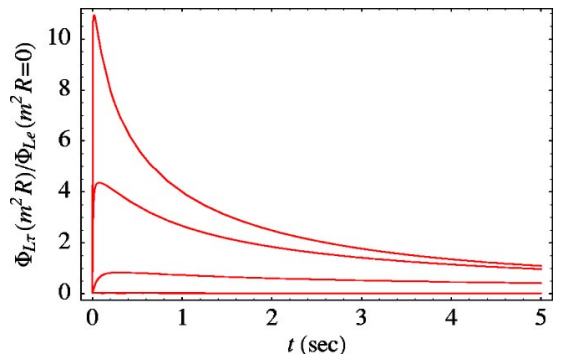

FIG. 5. The tau lepton number flux vs time during the first $5 \mathrm{sec}$, for increasing values of $m^{2} R=10^{-(12,10,8,6)}$, from bottom to top. The normalization is given by the value of the electron lepton number flux in the absence of new physics.

$m^{2} R$. At the same time, the total amount of drained energy increases significantly with $m^{2} R$, as a consequence of the faster cooling of the core. However, balancing the two effects, what is important is that the reduction of the portion emitted into the visible channel is always limited to $\sim 20 \%$ of the standard case, which is well acceptable in the light of the need of accounting for the SN 1987A signal: no direct upper bound on the parameter $m^{2} R$ then needs to be imposed.

The other significant result is the emission of a net tau lepton number, counterbalancing the tau antineutrinos that escape into the bulk. The emission can grow up to values $\sim 10$ times larger than the corresponding $\nu_{e}$ flux, for $m^{2} R$ at the end of the considered range. As for its time distribution, Fig. 5 shows that the $\nu_{\tau}$ emission is concentrated in the very first seconds. An analogous behavior is exhibited by the total energy emission, so that we can foresee a peculiar time dependence of the final neutrino number flux on Earth, more peaked at earlier times. Letting aside this general expectation, we focus in the following on time-integrated quantities.

Taking our outputs of the modified core evolution as the starting point, we now want to outline the subsequent vicissitudes and the ultimate fate of the neutrinos from the cooling phase in order to derive the possible consequences both for supernova physics and for the detectable signal on Earth.

First is the issue of how the energy flux and the sizeable $\nu_{\tau}$ net flux emitted from the inner core are redistributed by the subsequent diffusion that takes place up to the neutrino sphere (after which the neutrinos stream freely). A detailed examination of this phase would require studying the evolution of the mantle also; see, e.g., Ref. [14]. What we do is to assume that the energy $E$ emitted from the inner core ends almost equiparted in neutrinos and antineutrinos of all flavors, once the portion carried by the lepton number excesses has been subtracted. On the other hand, the (positive) lepton numbers $L_{e}$ and $L_{\tau}$ are separately conserved in the reprocessing. With these ingredients one can then determine the neutrino and antineutrino number fluxes in each flavor: $n_{\bar{\nu}_{j}}$ $\simeq 1 /\left\langle E_{\bar{\nu}_{j}}\right\rangle\left(E-\Sigma_{i}\left\langle E_{\nu_{i}}\right\rangle L_{i}\right) /\left(3+\Sigma_{i}\left\langle E_{\nu_{i}}\right\rangle /\left\langle E_{\bar{\nu}_{i}}\right\rangle\right), \quad n_{\nu_{j}} \simeq L_{j}$ $+n_{\nu_{j}}$, where $i, j=e, \mu, \tau$. For the average energies of the different families we use the typical values $\left\langle E_{\nu_{e}}\right\rangle$

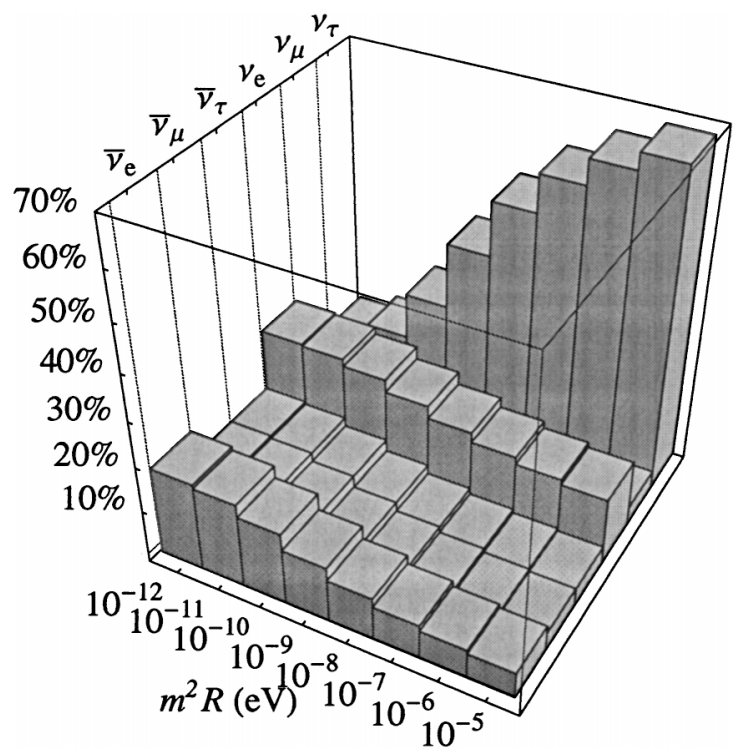

FIG. 6. Indicative composition of the neutrino flux leaving the neutrino spheres as a function of $m^{2} R$.

$\approx 13 \mathrm{MeV},\left\langle E_{\bar{\nu}_{e}}\right\rangle \approx 16 \mathrm{MeV}$ and $\left\langle E_{\nu_{\mu, \tau}, \bar{\nu}_{\mu, \tau}}\right\rangle \approx 23 \mathrm{MeV} .^{5} \mathrm{It}$ has recently been pointed out [15] that the mean energies of muon and tau neutrino and antineutrino can be closer to the electron antineutrino's, at the level of less than $1.2\left\langle E_{\bar{\nu}_{e}}\right\rangle$ during the core cooling phase. Nevertheless, such a variation does not change our analysis. We will comment on this issue later on.

Thus, coming out from the neutrino spheres, the flux displays a peculiar composition (see Fig. 6): for large values of $m^{2} R$, the $\nu_{\tau}$ 's are $\sim 5$ times more than in the case without new physics, at the expense of the $\nu_{e}$ 's and of all flavors of antineutrinos (all reduced by up to $\sim 60 \%$ ). These reductions, although not so large, go into the direction opposite to the need of rejuvenating the stalling shock wave and thus helping the explosion, since muon and tau neutrinos are much less efficient than the electron ones in transferring their energy to the lingering matter. On the other hand, this qualitative argument does not imply a strong drawback. The actual explosion mechanism may be hidden in the complexity of the system, whose simulation is still a nontrivial task.

Next, neutrinos and antineutrinos undergo the matter flavor oscillation in the peripheric low-density region of the star and the vacuum flavor oscillations in the journey from the supernova to Earth. We illustrate the outcome in the case of normal neutrino mass hierarchy. As for the oscillation parameters, we use $\theta_{23} \simeq 45^{\circ}$ [4] and $\theta_{12} \simeq 32^{\circ}$ [16], while we consider values for $\theta_{13}$ compatible with the Chooz bound [17]. The first transition at very high densities is produced by the small matter potential difference between tau and muon neutrinos, which is due to the mass difference of the corresponding leptons. This transition is found to be completely adiabatic and switches the $\stackrel{(-)}{\nu}_{\mu}, \stackrel{(-)}{\nu}_{\tau}$ flavor eigenstates to the

\footnotetext{
${ }^{5} \mathrm{We}$ are assuming that the average energies of the neutrinos at the neutrino sphere are not affected by the new physics effect, see [9].
} 

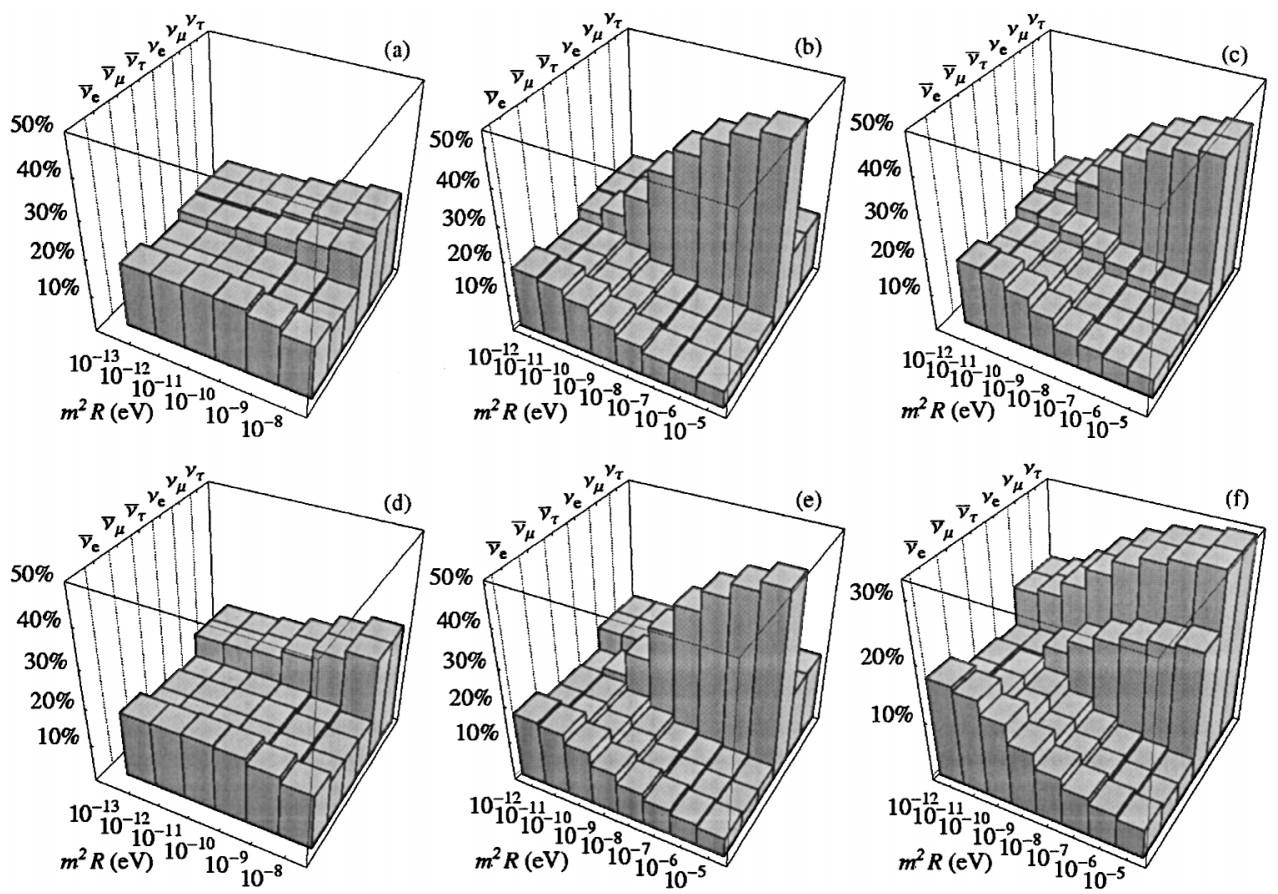

states rotated by the angle $\theta_{23}$, labeled $\stackrel{(-)}{\nu}_{\mu^{\prime}}$ and $\stackrel{(-)}{\nu}_{\tau^{\prime}}$. At lower densities, two more resonances are met, due to the charged current contribution to the electron matter potential. In the case of normal hierarchy, these resonances are both active in the neutrino sector. The $\nu_{e^{-}} \nu_{\mu}$, resonance turns out to be adiabatic under our assumptions while for the $\nu_{e}-\nu_{\tau^{\prime}}$ we consider a level crossing probability $P_{H}$, which can vary from 0 to 1 depending on the value of $\theta_{13}$. For a more detailed analysis, we refer the reader to [18]. Finally, neglecting terms proportional to $\sin \theta_{13}$, the neutrino and antineutrino fluxes $F$ reaching the Earth's surface are related to the neutrino sphere fluxes $F^{0}$ by the simple relations

$$
\begin{aligned}
F_{e}= & P_{H} s_{12}^{2} F_{e}^{0}+c_{12}^{2} F_{\mu}^{0}+\left(1-P_{H}\right) s_{12}^{2} F_{\tau}^{0} \\
F_{\mu}= & {\left[P_{H} c_{12}^{2} c_{23}^{2}+\left(1-P_{H}\right) s_{23}^{2}\right] F_{e}^{0}+s_{12}^{2} c_{23}^{2} F_{\mu}^{0} } \\
& +\left[P_{H} s_{23}^{2}+\left(1-P_{H}\right) c_{12}^{2} c_{23}^{2}\right] F_{\tau}^{0} \\
F_{\tau}= & {\left[P_{H} c_{12}^{2} s_{23}^{2}+\left(1-P_{H}\right) c_{23}^{2}\right] F_{e}^{0}+s_{12}^{2} s_{23}^{2} F_{\mu}^{0} } \\
& +\left[P_{H} c_{23}^{2}+\left(1-P_{H}\right) c_{12}^{2} s_{23}^{2}\right] F_{\tau}^{0} \\
F_{\bar{e}}= & c_{12}^{2} F_{\bar{e}}^{0}+s_{12}^{2} F_{\bar{\tau}}^{0} \\
F_{\bar{\mu}}= & s_{12}^{2} c_{23}^{2} F_{\bar{e}}^{0}+s_{23}^{2} F_{\mu}^{0}+c_{12}^{2} c_{23}^{2} F_{\bar{\tau}}^{0} \\
F_{\bar{\tau}}= & s_{12}^{2} s_{23}^{2} F_{\bar{e}}^{0}+c_{23}^{2} F_{\bar{\mu}}^{0}+c_{12}^{2} s_{23}^{2} F_{\bar{\tau}}^{0}
\end{aligned}
$$

where $s_{i j}$ and $c_{i j}$ are the sine and cosine of the angle $\theta_{i j}$.

The indicative final composition of the flux reaching the surface of the Earth is illustrated in Figs. 7(c) and 7(f) for the boundary values $P_{H}=1$ and $P_{H}=0$, corresponding respec-
FIG. 7. Indicative composition of the neutrino flux reaching the Earth's surface, as a function of $m^{2} R$, for the different cases of escape into the bulk: $\nu_{e}$ (first column), $\nu_{\mu}$ (second column), $\nu_{\tau}$ (third column); for $P_{H}=1$ (first line) and for $P_{H}=0$ (second line). tively to $\sin ^{2} \theta_{13} \lesssim 10^{-5}$ and $\sin ^{2} \theta_{13} \gtrsim 10^{-3}$. In the first case, the strong $\nu_{\tau}$ excess is distributed between muon and tau neutrinos, while the electron fraction decreases. The situation is different in the adiabatic $\left(P_{H}=0\right)$ case, where we expect a higher $\nu_{e}$ component. A general important feature is the $\bar{\nu}_{e}$ flux reduction that can amount to up to $\sim 60 \%$. Given the limited statistics of the 1987 event, the overall uncertainties on the expected neutrino fluxes (as predicted by full simulations, also based on assumptions on the progenitor star) and finally the approximate nature of the supernova core evolution adopted here, such a reduction is still compatible with present observations. Nevertheless, this feature is an interesting and potentially challenging one, and deserves to be addressed more closely in case of a future SN event with higher statistics. A similar remark also holds for the time structure of the neutrino signal mentioned above.

Our analysis has been limited so far to the case of tau neutrinos, but can be readily extended to the case of mixing of muon neutrinos with the bulk fermions. Indeed, no relevant modifications are needed to the model of evolution described in Sec. III, so that the general conclusions reached for tau neutrinos are still valid. In particular, the indicative fluxes coming out of the neutrino spheres are given in first approximation by a plot similar to the one in Fig. 6, with the excess in the $\nu_{\mu}$ channel. In turn, using Eqs. (6) and Eqs. (7), one can determine the compositions reaching the Earth's surface shown in Figs. 7(b) and 7(e); the value of $P_{H}$ is only marginally important in this case.

Finally, we can recall the results of [9] for the case of the electron neutrinos oscillating into the bulk and derive the compositions in Figs. 7(a) and 7(d) following the same steps.

Based on the above results, one can easily point out the most important observable modifications of the expected flux in a detector. First of all, as the net effect is always an increase in the total emitted lepton number, a crucial signature 
is the enhanced ratio between neutrinos and antineutrinos. This is a general feature of supernova neutrino oscillations into extra dimensions that does not depend on which flavor has a significant mixing with bulk neutrinos. What is case dependent is which channel the enhancement shows up in. First one can focus on the electron channel, which will have high statistics in the future supernova events. Indeed, $\bar{\nu}_{e}$ are the dominant signal in the present and in most of the future detectors, while $\nu_{e}$ can be efficiently collected by large Čerenkov detectors, already existing (SK, SNO) or proposed (UNO), and liquid-argon detectors (Icarus, LANNDD) [19]. For several cases in Fig. 7 the $\nu_{e} / \bar{\nu}_{e}$ ratio is significantly enlarged. However, it is also clear from Fig. 7 that, depending on the value of $P_{H}$ (i.e. of $\theta_{13}$ ), the effect of $\nu_{e}$ or $\nu_{\tau}$ conversion into the bulk could turn out to be irrelevant for the $\nu_{e} / \bar{\nu}_{e}$ channel in some cases, so that it would be necessary to measure the $\nu_{\mu}$ or $\nu_{\tau}$ fluxes. This is a harder task, which is however feasible at SNO, future detectors (UNO, LANNDD, OMNIS) [19] or even at large scintillator detectors (KamLAND, Borexino [20]). On the other hand, once an anomalous neutrino/antineutrino ratio is measured, how is it possible to distinguish which flavor does mix with the bulk? The answer lies in the energy spectrum. In fact, since the electron neutrinos are less energetic than the muon and tau ones when they leave the neutrino spheres, the effect of the $\nu_{e} \leftrightarrow \nu_{\mu, \tau}$ oscillation is to harden the $\nu_{e}$ spectrum on the Earth. The conversion into the bulk of the electron flavor, increasing the $\nu_{e}$ component flowing in the SN mantle, reduces this hardness, while the muon or tau conversion enhances it.

We have illustrated our results for a specific point in the parameter space of the standard neutrino oscillations. The residual dependence on $\theta_{12}$ (and $\theta_{23}$ ) is mild. On the other hand, the distribution of the neutrino flux enhancement in the three flavors depends significantly on the neutrino mass pattern. Disentangling the flavor structure of the mixing with bulk neutrinos would therefore require the knowledge of $\operatorname{sign}\left(\Delta m_{23}^{2}\right)[21]$.

As already mentioned, another source of uncertainty is the precise value of the mean energies of the neutrinos coming out from their neutrino spheres. We checked that the adoption of a $\left\langle E_{\nu_{\mu, \tau}, \bar{v}_{\mu, \tau}}\right\rangle$ closer to $\left\langle E_{\bar{\nu}_{e}}\right\rangle$, as suggested in [15], yields only minor modifications of our result; namely, each column in Fig. 6 and Fig. 7 is affected at most by a variation of $5-10 \%$ in composition, within the uncertainties of our simplified model. Thus, the analysis is unchanged and the general trend confirmed: we only remark that the closer the mean energies, the smaller the differences among the channels, although preserving the features described above.

Finally, in order to predict the actual signal in the detectors, one should also consider matter-induced oscillations inside the Earth. This effect has the power of dramatically changing the expected signal, but of course depends on the geographical position of the detectors at the time of arrival of the signal. For a given path in the Earth, it is not difficult to include the matter effect (see $[22,18])$.

While we have so far considered the case in which the neutrino escape is dominated by the oscillations in the bulk of one of the three neutrinos, it is also possible that all SM neutrinos are involved in the energy loss. In this case, the neutrino flux on the Earth would still be enhanced over the antineutrino one. However, the neutrino flux composition would also be determined by the relative size of the three mixing parameters $m_{e}, m_{\mu}, m_{\tau}$. The evolutions of the three neutrino abundances in the core would be coupled through the temperature and the matter potential. The feedback mechanisms would however still take place, but we expect the $m_{i}-R$ parameter space for the three channels to be slightly reduced.

\section{CONCLUSIONS}

We dealt with the mixing of the Standard Model neutrinos with bulk sterile fermion fields, a natural and general feature of models with extra dimensions. In particular, we focused on the muon and tau case, while the electron case was addressed in [9]. This mixing provides an unconventional escape channel for neutrinos in the supernova core during the cooling phase, which could in principle give strong bounds on the parameters of the extra dimensions when facing the observed SN 1987A signal. In order to simplify the core dynamics, we restricted the analysis to the case of $\nu_{\tau}$, but we easily completed it to the case of $\nu_{\mu}$.

Given the sign of the matter potential, the process consists in the escape of tau antineutrinos into the bulk, acting in parallel with diffusion. We showed that a feedback mechanism turns on, preventing extreme and unacceptable modifications of the evolution to occur (in particular, the characteristic time scale of deleptonization and cooling, of order 10 sec, is preserved). The direct bound on the parameters of extra dimensions can then be relaxed by about 4 orders of magnitude. The upper limit on the relevant combination $m^{2} R$ is lifted from $10^{-10} \mathrm{eV}$ to $5 \times 10^{-5} \mathrm{eV}$, the new limit now coming from subleading effects. In Fig. 1, we showed the allowed region in the $m-R$ plane. The feedback is mainly due to the building up of a degenerate sea of trapped $\nu_{\tau}$ and thus of a positive tau neutrino chemical potential that suppresses the number of $\bar{\nu}_{\tau}$ 's candidate to escape. For comparison, in the $\nu_{e}\left(\right.$ and $\bar{\nu}_{e}$ ) case in [9] this kind of feedback acts together with the feedback on the matter potential in a sizable portion of the core.

Although in such a "safe" way, the supernova core evolution is significantly affected by the escape process, and we studied the main modifications. First of all, the cooling is sped up with respect to the standard case. Second, a sizable net tau lepton number is emitted by the core, concentrated in the very first seconds. Therefore a neutrino number flux more peaked at earlier times can be predicted.

Next we speculated on the phenomenological consequences on the revival of the shock and on the neutrino signal reaching the Earth. As for the first point, since the $\nu_{e}$ flux is lowered by the reshuffling of the neutrino fluxes (although not largely), we suggested that the $\nu_{\mu}, \nu_{\tau}$ escape may reduce the energy transfer to matter and thus the push to the explosion. As for the second point, the compatibility with the SN 1987A signal is preserved once one is willing to accept a 
certain reduction of the $\bar{\nu}_{e}$ component. Collecting the results for the $\nu_{e}, \nu_{\mu}$ and $\nu_{\tau}$ escape cases, we indicated as signatures, in cases of future galactic SN events, a general dominance of neutrino flux over antineutrinos and, depending on the flavor that mixes with bulk fields, some peculiar structures of the relative enhancements in the neutrino fluxes and a harder or softer $\nu_{e}$ spectrum.

\section{ACKNOWLEDGMENTS}

This work has been partially supported by MIUR and by the EU under TMR contract HPRN-CT-2000-00148. Part of the work of A.R. was done while at Scuola Normale Superiore. M.C. acknowledges the hospitality of the CERN Theory Division, where this work was completed.
[1] K.R. Dienes, E. Dudas, and T. Gherghetta, Nucl. Phys. B557, 25 (1999); N. Arkani-Hamed, S. Dimopoulos, G.R. Dvali, and J. March-Russell, Phys. Rev. D 65, 024032 (2002); G.R. Dvali, and A.Y. Smirnov, Nucl. Phys. B563, 63 (1999).

[2] For general reviews and extensive references, see: H.A. Bethe, Rev. Mod. Phys. 62, 801 (1990); G.G. Raffelt, Stars as Laboratories for Fundamental Physics: The Astrophysics of Neutrinos, Axions, and Other Weakly Interacting Particles (Chicago University Press, Chicago, 1996); J.M. Lattimer, in Nuclear Equation of State, edited by A. Ansari and L. Satpathy (World Scientific, Singapore, 1996), pp. 83-208; M. Prakash, J.M. Lattimer, J.A. Pons, A.W. Steiner, and S. Reddy, Lect. Notes Phys. 578, 364 (2001); H.T. Janka, K. Kifonidis, and M. Rampp, ibid. 578, 333 (2001).

[3] SNO Collaboration, Q.R. Ahmad et al., Phys. Rev. Lett. 87, 071301 (2001); 89, 011301 (2002); 89, 011302 (2002).

[4] Super-Kamiokande Collaboration, Y. Fukuda et al., Phys. Rev. Lett. 81, 1562 (1998).

[5] A. Lukas and A. Romanino, hep-ph/0004130; A. Lukas, P. Ramond, A. Romanino, and G.G. Ross, Phys. Lett. B 495, 136 (2000).

[6] KAMIOKANDE-II Collaboration, K. Hirata et al., Phys. Rev. Lett. 58, 1490 (1987); R.M. Bionta et al., ibid. 58, 1494 (1987).

[7] R. Barbieri, P. Creminelli, and A. Strumia, Nucl. Phys. B585, 28 (2000).

[8] A. Lukas, P. Ramond, A. Romanino, and G.G. Ross, J. High Energy Phys. 04, 010 (2001) .

[9] G. Cacciapaglia, M. Cirelli, Y. Lin, and A. Romanino, Phys. Rev. D 67, 053001 (2003).

[10] S. Hannestad, H.T. Janka, G.G. Raffelt, and G. Sigl, Phys. Rev. D 62, 093021 (2000).

[11] I. Antoniadis, Phys. Lett. B 246, 377 (1990); J.D. Lykken, Phys. Rev. D 54, 3693 (1996); N. Arkani-Hamed, S. Dimopoulos, and G.R. Dvali, Phys. Lett. B 429, 263 (1998); I. Antoniadis, N. Arkani-Hamed, S. Dimopoulos, and G.R. Dvali, ibid. 436, 257 (1998).

[12] N. Arkani-Hamed, S. Dimopoulos, and G.R. Dvali, Phys. Rev. D 59, 086004 (1999); T. Han, J.D. Lykken, and R.J. Zhang, ibid. 59, 105006 (1999); S. Cullen and M. Perelstein, Phys. Rev. Lett. 83, 268 (1999); C. Hanhart, D.R. Phillips, S. Reddy, and M.J. Savage, Nucl. Phys. B595, 335 (2001); C. Hanhart,
J.A. Pons, D.R. Phillips, and S. Reddy, Phys. Lett. B 509, 1 (2001); L.J. Hall and D.R. Smith, Phys. Rev. D 60, 085008 (1999); S. Hannestad, ibid. 64, 023515 (2001).

[13] S. Reddy, M. Prakash, and J.M. Lattimer, Phys. Rev. D 58, 013009 (1998).

[14] A. Mezzacappa, M. Liebendorfer, O.E. Messer, W.R. Hix, F.K. Thielemann, and S.W. Bruenn, Phys. Rev. Lett. 86, 1935 (2001); A. Burrows, T. Young, P. Pinto, R. Eastman, and T. Thompson, astro-ph/9905132; G.G. Raffelt, Astrophys. J. 561, 890 (2001); M.T. Keil, G.G. Raffelt, and H.T. Janka, astro-ph/0208035.

[15] See M.T. Keil, G.G. Raffelt, and H.T. Janka in Ref. [14].

[16] KamLAND Collaboration, K. Eguchi et al., Phys. Rev. Lett. 90, 021802 (2003); V. Barger and D. Marfatia, Phys. Lett. B 555, 144 (2003); G.L. Fogli, E. Lisi, A. Marrone, D. Montanino, A. Palazzo, and A.M. Rotunno, Phys. Rev. D 67, 073002 (2003); M. Maltoni, T. Schwetz, and J.W. Valle, ibid. 67, 093003 (2003); P. Creminelli, G. Signorelli, and A. Strumia, J. High Energy Phys. 05, 052 (2001); see addendum in hep-ph/0102234v4; A. Bandyopadhyay, S. Choubey, R. Gandhi, S. Goswami, and D.P. Roy, Phys. Lett. B 559, 121 (2003); J.N. Bahcall, M.C. Gonzalez-Garcia, and C. Peña-Garay, J. High Energy Phys. 02, 009 (2003); H. Nunokawa, W.J. Teves, and R. Zukanovich Funchal, Phys. Lett. B 562, 28 (2003); P. Aliani, V. Antonelli, M. Picariello, and E. Torrente-Lujan, hep-ph/0212212; P.C. de Holanda and A.Y. Smirnov, J. Cosmol. Astropart. Phys. 02, 001 (2003).

[17] M. Apollonio et al., Eur. Phys. J. C 27, 331 (2003).

[18] E.K. Akhmedov, C. Lunardini, and A.Y. Smirnov, Nucl. Phys. B643, 339 (2002).

[19] F. Cei, Int. J. Mod. Phys. A 17, 1765 (2002).

[20] J.F. Beacom, W.M. Farr, and P. Vogel, Phys. Rev. D 66, 033001 (2002).

[21] See, for instance, M. Apollonio et al., hep-ph/0210192.

[22] A.Y. Smirnov, D.N. Spergel, and J.N. Bahcall, Phys. Rev. D 49, 1389 (1994); B. Jegerlehner, F. Neubig, and G. Raffelt, ibid. 54, 1194 (1996); A.S. Dighe and A.Y. Smirnov, ibid. 62, 033007 (2000); M. Kachelriess, R. Tomas, and J.W. Valle, J. High Energy Phys. 01, 030 (2001); M. Kachelriess, A. Strumia, R. Tomas, and J.W. Valle, Phys. Rev. D 65, 073016 (2002); C. Lunardini and A.Y. Smirnov, Nucl. Phys. B616, 307 (2001). 